\title{
Evaluación farmacocinética/farmacodinámica (PK/PD) de un esquema de administración oral de metronidazol en intervalo ampliado para el manejo de infecciones producidas por Bacteroides fragilis
}

\author{
Felipe Morales-León, Carlos von Plessing-Rossel, Lorenzo Villa-Zapata, Pola Fernández-Rocca, \\ Cindy Sanhueza-Sanhueza, Helia Bello-Toledo y Sergio Mella-Montecinos
}

\section{Pharmacokinetics/pharmacodinamic (PK/PD) evaluation of a short course of oral administration of metronidazole for the management of infections caused by Bacteroides fragilis}

Introduction: Metronidazole is the antibiotic of choice for the management of infections caused by anaerobes. Its administration requires multiple daily doses causing increased medication errors. Due to its high post-antibiotic effect and rapid concentration-dependent bactericidal activity, administration of this antibiotic in an extended dosing interval would achieve PK/PD parameters effectively. Objective: To assess the probability of achieving effective PK/PD relationship with the administration of 1,000 mg every 24 hours of metronidazole for Bacteroides fragilis infections. Methods: A clinical trial was conducted in a group of volunteers who received a single oral dose of 500 or $1,000 \mathrm{mg}$ of metronidazole. Determinations of values of $\mathrm{C}_{\max }, \mathrm{t}_{\max }$, and $\mathrm{AUC}_{0-24 \mathrm{~h}}$ determined using the trapezoidal method, were obtained for a Markov simulation that would allow for determining the likelihood of achieving a $\mathrm{AUC}_{0-24 \mathrm{~h}} / \mathrm{MIC}$ ratio above 70 for infections caused by susceptible B. fragilis. Results: $\mathrm{C}_{\max }(24,03$ $\pm 6,89 \mathrm{mg} / \mathrm{L})$ and $\mathrm{t}_{\max }(1,20 \pm 0.80 \mathrm{hrs})$ and the value of $\mathrm{AUC}_{0-24 \mathrm{~h}}(241.91 \pm 48.14 \mathrm{mg} * \mathrm{~h} / \mathrm{L})$ were determined. The probability of obtaining a $\mathrm{AUC}_{0-24} / \mathrm{MIC}$ ratio greater than 70 was greater than $99 \%$. Conclusion: From a pharmacokinetic perspective, with the administration of a daily dose of $1,000 \mathrm{mg}$ of metronidazole, it is possible to achieve a therapeutic goal of $\mathrm{AUC}_{0-24 \mathrm{~h}} / \mathrm{MIC}$ ratio above 70 for the treatment of anaerobic infections.

Key words: Metronidazole, PK/PD, Bacteroides fragilis, Markov, AUC/MIC.

Palabras clave: Metronidazol, PK/PD, Bacteroides fragilis, Markov, AUC/CIM.

\section{Introducción}

$\mathrm{L}$ as infecciones anaeróbicas son una causa común de cuadros potencialmente peligrosos con una elevada tasa de mortalidad. Éstos van desde infecciones periodontales hasta neumonías, bacteriemias y abscesos potencialmente mortales. Se estima que alrededor de $5 \%$ de los cuadros de bacteriemias en adultos son provocadas por bacterias anaerobias, especialmente por especies de los géneros Clostridium, Fusobacterium, Peptostreptococcus y principalmente por cepas de Bacteroides fragilis, las que provienen de infecciones intra-abdominales, pélvicas, de piel y tejidos blandos ${ }^{1-3}$.

Por su parte, metronidazol es el antimicrobiano de elección para el manejo de infecciones sistémicas producidas por B. fragilis ${ }^{4}$. Este antimicrobiano exhibe un efecto bactericida rápido dependiente de la concentración además de un prolongado efecto post antibiótico (EPA) sobre bacterias anaeróbicas sensibles, el que puede ser superior a tres horas 5 . Adicionalmente, este efecto se puede prolongar al mantenerse una concentración del antimicrobiano bajo la concentración inhibitoria mínima (CIM) del microorganismo ${ }^{6}$. Este efecto de supresión persistente del crecimiento microbiano ha sido explicado por diversos fenómenos, pudiendo mencionarse, por ejemplo, el desarrollo de ciertos cambios morfológicos que se producen en los microorganismo, el efecto post-CIM y efecto post-antibiótico estimulador de leucocitos (EPAEL) que para metronidazol, es equivalente a la concentración encontrada en fluidos extracelulares ${ }^{5}$. Adicionalmente, y desde el punto de vista farmacodinámico (PD), el parámetro farmacocinético/farmacodinámico $(\mathrm{PK} / \mathrm{PD})$ que mejor describe la eficacia de la actividad bactericida de metronidazol es la relación entre área bajo la curva $\left(\mathrm{AUC}_{0-24 \mathrm{~h}}\right)$ y la CIM del microorganismo?

En la actualidad, para el manejo de las infecciones anaerobias, se requiere de la administración habitual de dosis bajas de metronidazol, con elevada frecuencia. Esta
Universidad de Concepción Concepción, Chile. Facultad de Farmacia, Departamento de Farmacia Farmacia Clínica (FM-L, PF-R). Laboratorio de Biofarmacia (CvP-R) Laboratorio de Investigación en Agentes Antibacterianos (LIAA) (HB-T)

Facultad de Medicina, Departamento de Medicina Interna (SM-M).

The University of Arizona, Arizona, USA.

Department of Pharmacy Practice and Science

Pharmaceutical Economics Policy and Outcomes University of Concepcion, College of Pharmacy, Chile (LV-Z).

Hospital Penco-Lirquén, Concepción, Chile.

Unidad de Farmacia (CS-S).

Establecimiento donde se realizó el estudio:

Departamento de Farmacia,

Facultad de Farmacia, Universidad de Concepción, Chile.

Sergio Mella Montecinos declara recibir pago de honorarios por conferencias de Merk, Sharp \& Dohme (MSD) y el apoyo para viajes por parte de AstraZeneca y MSD. El resto de los autores declara no presentar conflictos de intereses.

Fuente de financiamiento: Proyecto DIUC No 212.074.049-1.0; Universidad de Concepción.

Recibido: 18 de junio de 2014 Aceptado: 17 de noviembre de 2014

Correspondencia a: Felipe Eduardo Morales-León felimora@udec.cl 
situación genera numerosos problemas, ya que al incrementar el número de administraciones diarias se favorece la falta de adherencia al tratamiento, el incremento en la probabilidad que ocurran errores de administración y por tanto, un aumento en la incidencia de efectos adversos indeseados. De tal forma que, considerando las propiedades PD, el EPA junto a la actividad bactericida dependiente de la concentración señala que la dosificación óptima de este antimicrobiano en infecciones anaeróbicas correspondería a aquella en la cual se administren dosis más elevadas, con menor frecuencia. En este trabajo, se determinó la probabilidad aleatoria de alcanzar una relación $\mathrm{PK} / \mathrm{PD}$ terapéuticamente efectiva con la administración diaria de metronidazol oral en una dosis de $1.000 \mathrm{mg}$ cada $24 \mathrm{~h}$.

\section{Métodos}

\section{Diseño del estudio}

Se realizó un ensayo clínico controlado, cruzado, aleatorio simple, sobre un grupo de 14 voluntarios adultos sanos que cumplieran con los requisitos de inclusión. Cada uno de ellos recibió la administración oral de una única dosis de metronidazol de $500 \mathrm{mg}$ ó $1.000 \mathrm{mg}$. Los voluntarios fueron enrolados e invitados a participar de este estudio por medio de la entrega del correspondiente consentimiento informado. Posteriormente, fueron divididos de forma aleatoria en dos grupos. El grupo A recibió una única dosis de $500 \mathrm{mg}$ mientras que el grupo B recibió una dosis experimental de $1.000 \mathrm{mg}$. Una vez finalizada la etapa de recolección de muestras y transcurridas $24 \mathrm{~h}$ desde la administración del fármaco, se procedió a establecer un período mínimo de 7 días de ventana, donde los voluntarios no utilizaron medicamento alguno. Con posterioridad, se invirtió el orden del grupo de estudio inicial y se procedió de la forma descrita anteriormente. Las dosis administradas fueron realizadas en comprimidos de metronidazol (Metropast ${ }^{\mathrm{B}}$, Laboratorio Pasteur $^{\circledR}$ ) de $500 \mathrm{mg}$ cada uno, pertenecientes al mismo lote de producción.

\section{Aspectos éticos}

Se incluyó en este estudio a voluntarios mayores de 18 años y menores a 60 años, de cualquier género, y que tuvieran un índice de masa corporal (IMC) calculado no superior a $28 \mathrm{~kg} / \mathrm{m}^{2}$. Adicionalmente, se excluyó de este estudio a todo voluntario portador de enfermedades crónicas descompensadas, con antecedentes de falla renal aguda $(\mathrm{Cl}$ creatinina $<10 \mathrm{~mL} / \mathrm{min}$ calculado según Cockcroft-Gault), consumidor crónico de alcohol y/o embarazo o sospecha de embarazo. Cada voluntario fue enrolado e invitado a participar de este estudio por medio de la entrega del correspondiente consentimiento informado. El protocolo de investigación fue aprobado por los Comités de Ética de la Universidad de Concepción y del Servicio de Salud de Concepción, Chile.

\section{Metodología analítica}

La determinación de las concentraciones plasmáticas de metronidazol fue realizada empleando un método analítico previamente validado, basado en cromatografía líquida de alta resolución (HPLC) acoplado a detector de arreglo de diodos (DAD). Se empleó como base el método propuesto por Do Nascimento y cols. ${ }^{8} \mathrm{Se}$ utilizó un cromatógrafo (Merck Hitachi ${ }^{\circledR}$ modelo L-7100) acoplado a un detector DAD (Merck Hitachi ${ }^{\circledR}$ modelo L-7200). Las separaciones analíticas se realizaron con una columna C-18 de 250 por 4,6 mm (Kromasil ${ }^{\circledR}$ ) y una fase móvil compuesta por tampón fosfato $(0,01 \mathrm{M} ; \mathrm{pH}$ 4,7)/Acetonitrilo (Merck LiChrosolv ${ }^{\circledR}$ ) en proporción $80 / 20$ v/v. Para la validación del método se empleó un estándar grado HPLC de metronidazol (Sigma-Aldrich FlukaVetranal $^{\circledR}$ ). Se obtuvieron muestras de sangre total de voluntarios sanos que habían recibido una dosis de metronidazol. Las muestras fueron obtenidas utilizando tubos con heparina de litio (Vacutainer $\mathrm{DB}^{\circledR}$ ) de $2 \mathrm{~mL}$, los que fueron centrifugadas (3500 rpm) para obtener plasma. Las proteínas fueron precipitadas con una solución de $\mathrm{HClO}_{4}$ 0,5M (Merck Suprarpur ${ }^{\circledR}$ ) en proporción 1:1 y filtradas por membrana de HAWP Millipore ${ }^{\circledR}(0,45 \mu \mathrm{m})$. Se evaluó la linealidad en un intervalo de concentración de 0,25 a $35 \mathrm{mg} / \mathrm{mL}$ para obtener un modelo de respuesta lineal $(\mathrm{r}=0,99973)$. Se calcularon además los límites de detección $(0,10 \mathrm{mg} / \mathrm{L})$, límites de cuantificación $(0,3 \mathrm{mg} /$ $\mathrm{mL}$ ), precisión intermedia y repetitividad.

\section{Determinación de parámetros farmacocinéticos}

Se realizó la toma de muestras sanguíneas por punción venosa, recolectando las muestras a intervalos de tiempo definidos: 0,3 - 0,5 - 1 - 1,3 - 2 - 3 - 6 - 8 - 12 - 18 y $24 \mathrm{~h}$ después de la administración del fármaco. Todas estas muestras fueron procesadas y cuantificadas con el método descrito anteriormente. Con estos datos, se construyeron los perfiles farmacocinéticos individuales a partir de la construcción de las curvas de concentración plasmática versus tiempo. A partir de la observación directa de las curvas, se obtuvieron los valores de $\mathrm{C}_{\max }$, $\mathrm{t}_{\max } \mathrm{y} \mathrm{C}_{\text {min }}$. Adicionalmente, se estimó el valor de $\mathrm{AUC}_{0-24 \mathrm{~h}}$ por medio del método trapezoidal y el $\mathrm{AUC}_{0-\infty}$ a partir de la extrapolación de los perfiles farmacocinéticos, desde tiempo cero al infinito, con la ayuda del software PK RStudio (GNU RStudio Versión 3.0.1). Adicionalmente

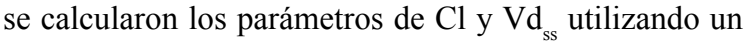
modelo no compartimental.

Se estableció la relación existente entre el $\mathrm{AUC}_{0-24 \mathrm{~h}} \mathrm{de}$ la administración de ambos esquemas y la CIM de Bacteroides fragilis según la relación de la Figura 1. Los valores de susceptibilidad para $B$. fragilis a metronidazol fueron 


\section{$\frac{P K}{P D}=\frac{A U C_{0-24 h}}{C I M}$}

Figura 1. Relación PK/PD que describe la efectividad de metronidazol.

obtenidos desde lo publicado por el European Committee on Antimicrobial Susceptibility Testing (EUCAST), de tal forma que se consideró como cepas susceptibles a aquellas cepas que tuvieran un valor de $\mathrm{CIM} \geq 2 \mathrm{mg} / \mathrm{mL}$ de metronidazol. ${ }^{9}$

\section{Análisis estadístico}

Todos los parámetros farmacocinéticos fueron sometidos al análisis estadístico empleando para ello la prueba t-student para muestras independientes, considerando aquellos resultados estadísticamente significativos con un valor de $\mathrm{p}<0,05$. Estos análisis fueron realizados con la ayuda del software estadístico STATA Versión 11 (StataCorp ${ }^{\circledR}$ ).

\section{Simulación de Montecarlo vía cadena de Markov}

Con el propósito de establecer la probabilidad de alcanzar la meta terapéutica de $\mathrm{AUC}_{0-24 \mathrm{~h}} / \mathrm{CIM}$ con ambos esquemas de administración, se realizó una simulación de los datos experimentales empleando el método de Montecarlo vía cadena de Markov (MCMC). Para ello, se consideró el parámetro experimental medio de $\mathrm{AUC}_{0-24 \mathrm{~h}}$ para dos esquemas simulados de administración de $1.000 \mathrm{mg}$ cada 24 h y $500 \mathrm{mg}$ cada $8 \mathrm{~h}$ junto con su respectivo coeficiente de variación $(\mathrm{CV})$. Estos parámetros fueron simulados a partir de los datos experimentales, considerando un comportamiento farmacocinético mono-compartimental con absorción y eliminación de primer orden. Adicionalmente, se consideró un valor de CIM de $2 \mathrm{mg} / \mathrm{mL}^{9}$ para $B$. fragilis. De esta forma, y considerando la base de un mínimo de 2.000 réplicas para cada uno de los parámetros, se estimó la probabilidad aleatoria que la relación PK/PD alcance valores comprendidos entre 20 y 70., 7,10,11. Estos cálculos fueron realizados por medio del software de análisis RStudio (GNU RStudio Versión 3.0.1).

\section{Resulltados}

Se reclutó a un total de 14 voluntarios quienes cumplieron con los criterios de inclusión y exclusión. Destacó una proporción mayor de voluntarios de sexo femenino (64\%), con una distribución homogénea según edad (voluntarios entre 23 y 27 años). Todos se encontraron en un rango de peso $(69,2 \mathrm{~kg})$ y talla $(1,70 \mathrm{~m})$ normal lo cual se reflejó en un valor medio de IMC de $24 \pm 2,4 \mathrm{~kg} /$ $\mathrm{m}^{2}$, con diferencias significativas en estos parámetros para ambos sexos (Tabla 1).

\section{Parámetros farmacocinéticos}

En la Tabla 2, se observan los parámetros farmacocinéticos determinados para cada esquema administrado. Es importante señalar que trascurridas $24 \mathrm{~h}$ desde la administración de una dosis de $500 \mathrm{mg}$ de metronidazol vía oral, las concentraciones plasmáticas mínimas $\left(\mathrm{C}_{\min }\right)$ fueron indetectables por lo cual no fue posible establecer su valor. Sin embargo, para la administración de una dosis de $1.000 \mathrm{mg}$ de metronidazol, este valor se situó en 2,5 \pm $0,7 \mathrm{mg} / \mathrm{mL}$. Respecto a la $\mathrm{C}_{\text {max }}$, destacaron valores de 11,9 $\pm 1,7 \mathrm{mg} / \mathrm{mL}$ y $24,0 \pm 6,9 \mathrm{mg} / \mathrm{mL}$ para la administración de una única dosis de $500 \mathrm{mg}$ y $1.000 \mathrm{mg}$, respectivamente. Ambas concentraciones fueron alcanzadas a 1,00 $\pm 0,40$ y $1,20 \pm 0,80 \mathrm{~h}$ después de su administración, respectivamente.

\begin{tabular}{|c|c|c|c|c|c|c|c|}
\hline & \multicolumn{2}{|c|}{$\begin{array}{l}\text { Hombre } \\
(n: 5)\end{array}$} & \multicolumn{2}{|c|}{$\begin{array}{l}\text { Mujer } \\
\text { (n: 9) }\end{array}$} & \multicolumn{2}{|c|}{$\begin{array}{l}\text { Total } \\
\text { (n: 14) }\end{array}$} & \multirow[t]{2}{*}{$p^{*}$} \\
\hline & Media & SD & Media & SD & Media & SD & \\
\hline Edad (años) & 25 & 4,3 & 25 & 4,1 & 25 & 4 & 0,999 \\
\hline Peso (kg) & 80,6 & 5,9 & 62,8 & 10,3 & 69,2 & 12,4 & $0,004^{*}$ \\
\hline Talla (m) & 1,76 & 0,04 & 1,66 & 0,08 & 1,70 & 0,08 & $0,024^{*}$ \\
\hline IMC $\left(\mathrm{kg} / \mathrm{m}^{2}\right)$ & 26 & 2 & 23 & 2 & 24 & 2,4 & $0,020^{*}$ \\
\hline
\end{tabular}

Tabla 2. Parámetros farmacocinéticos para la administración oral de metronidazol en monodosis de $500 \mathrm{mg}$ y $1.000 \mathrm{mg}$

\begin{tabular}{|c|c|c|c|c|c|}
\hline \multirow{2}{*}{$\begin{array}{l}\text { Parámetro } \\
\text { Med } \pm \text { SD } \\
\text { IC- } 95 \%\end{array}$} & \multicolumn{2}{|c|}{ Esquema $500 \mathrm{mg}(\mathrm{n}: 11)$} & \multicolumn{2}{|c|}{ Esquema $1.000 \mathrm{mg}$ ( $\mathrm{n:}$ 14) } & \multirow[t]{2}{*}{$\mathbf{p}^{*}$} \\
\hline & Med \pm SD & IC- $95 \%$ & & & \\
\hline$C_{\max }(\mathrm{mg} / \mathrm{mL})$ & $11,87 \pm 1,71$ & $10,72-13,02$ & $24,03 \pm 6,89$ & $20,05-28,01$ & $<0,05^{*}$ \\
\hline$C_{\min }(\mathrm{mg} / \mathrm{mL})$ & - & - & $2,53 \pm 0,65$ & $2,13-2,93$ & ND \\
\hline $\mathrm{t}_{\text {max }}$ (hrs) & $1,00 \pm 0,40$ & $0,73-1,27$ & $1,20 \pm 0,80$ & $0,74-1,66$ & 0,457 \\
\hline$A \cup C_{0-24 h}\left(h^{*} \mathrm{mg} / \mathrm{L}\right)$ & $109,15 \pm 17,10$ & $97,66-120,64$ & $241,91 \pm 48,14$ & $214,11-269,71$ & $<0,05^{*}$ \\
\hline$A \cup C_{0-\infty}(h * m g / L)$ & $118,83 \pm 24,90$ & $102,10-135,56$ & $268,11 \pm 49,67$ & $239,43-296,79$ & $<0,05^{*}$ \\
\hline$V d_{s s}\left(L^{*} k^{-1}\right)$ & $0,61 \pm 0,20$ & $0,48-0,74$ & $0,56 \pm 0,14$ & $0,48-0,64$ & 0,470 \\
\hline$t_{1 / 2}(h)$ & $6,11 \pm 1,32$ & $-6,70$ & 1,36 & $-8,24$ & $0,02^{*}$ \\
\hline $\mathrm{Cl}\left(\mathrm{L}^{*} \mathrm{~h}^{-1}\right)$ & $4,28 \pm 0,69$ & $3,82-4,74$ & $3,86 \pm 0,75$ & $3,43-4,29$ & 0,164 \\
\hline$A \cup C_{0-24 h} / M I C$ & $54,30 \pm 7,20$ & $48,83-60,32$ & $121,0 \pm 24,10$ & $107,0-134,90$ & $<0,05^{*}$ \\
\hline
\end{tabular}

*Prueba t-student para muestras independientes. Significativos aquellos valores con $p<0,05$; ND: no disponible. 
Al observar los valores del área bajo la curva (AUC), es importante destacar la diferencia estadísticamente significativa respecto a ambos esquemas de administración.

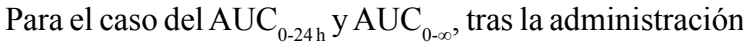
de una dosis de $500 \mathrm{mg}$ se alcanzaron valores de 109,15 $\pm 17,10$ y $118,83 \pm 24,90 \mathrm{~h} * \mathrm{mg} / \mathrm{mL}$, respectivamente, mientras que para la administración de $1.000 \mathrm{mg}$ en dosis única, estos parámetros se incrementaron proporcionalmente alcanzando $241,91 \pm 48,14$ y $268,11 \pm 49,67 \mathrm{~h} * \mathrm{mg} /$ $\mathrm{mL}$, respectivamente.

\section{Determinación de la relación PK/PD}

Como resultado de la simulación de los parámetros en estado estacionario, se determinó que tras la administración de un esquema habitual de metronidazol (500 mg cada $8 \mathrm{~h}$ ), no se alcanzó la meta terapéutica establecida ya que la relación $\mathrm{AUC}_{0-24 \mathrm{~h}} / \mathrm{CIM}$ fue de $54,3 \pm 7,2$. Por el contrario, tras la administración de un esquema ampliado de dosificación de $1.000 \mathrm{mg}$ cada 24 h sería posible alcanzar valores comprendidos entre 107 y 134,90, valores muy superior a la relación considerada como terapéuticamente efectiva.

\section{Simulación de Montecarlo vía cadena Markov}

En la Figura 2 se observa la representación de las probabilidades de alcanzar la relación PK/PD de cada esquema de administración. Según los resultados de la simulación, con la administración de un esquema de $500 \mathrm{mg}$ cada $8 \mathrm{~h}$, existe una probabilidad teórica de $98 \%$ para alcanzar una relación $\mathrm{PK} / \mathrm{PD}$ superior a 40, mientras que para la meta de $\mathrm{AUC}_{0-24 \mathrm{~h}} / \mathrm{CIM}$ de 70 , la probabilidad de alcanzarla con este esquema es inferior a $0,01 \%$. En contraste, para la

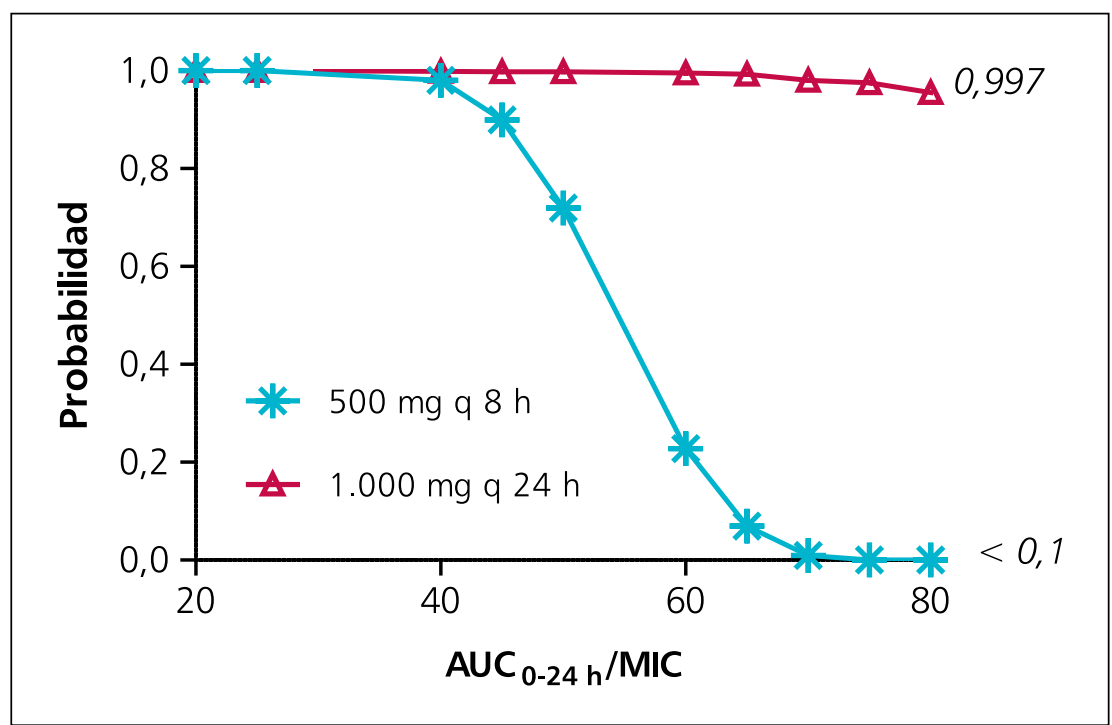

Figura 2. Probabilidad de alcanzar la meta terapéutica con la administración de 500 mg cada 8 h versus $1.000 \mathrm{mg}$ cada $24 \mathrm{~h}$. administración de un esquema de $1.000 \mathrm{mg}$ cada $24 \mathrm{~h}$ existe un $100 \%$ de probabilidad de alcanzar una relación PK/PD de 40 mientras que para la meta de 70, la probabilidad alcanzó a $98 \%$.

\section{Discusión}

En este estudio participó una mayor proporción de voluntarios de sexo femenino. Al respecto, el efecto que puede ejercer el sexo sobre las características farmacocinéticas se debe principalmente a diferencias en la composición corporal, como por ejemplo, las diferencias respecto a la talla e IMC, los cuales resultan mayores en hombres que en mujeres. Estos parámetros resultan en diferencias respecto al volumen de distribución $(\mathrm{Vd})$ y el clearance $(\mathrm{Cl})$, los que pueden ser levemente superior en hombres. Adicionalmente, y pese a estas consideraciones, no se observaron variaciones importantes respecto a los valores del $\mathrm{Cl}$ y $\mathrm{Vd}$ para ambos esquemas administrados.

Por su parte, destacó el incremento proporcional en $\mathrm{C}_{\max }$ siendo esta situación predecible ya que al tratarse de un fármaco con un comportamiento lineal respecto a su absorción, es esperable que al incrementar al doble la dosis administrada las concentraciones plasmáticas incremente proporcionalmente en el mismo sentido. Por su parte, los valores encontrados en nuestro estudio son superiores a los descritos por algunos autores ${ }^{12-14}$ quienes reportan valores de $\mathrm{C}_{\text {max }}$ para la administración de $500 \mathrm{mg}$ de $9,0 \mathrm{mg} / \mathrm{mL}$ y de $19,6 \mathrm{mg} / \mathrm{mL}$ para la administración de $1.000 \mathrm{mg}$. Adicionalmente, y según lo descrito por algunos autores $^{15-17}$, la $\mathrm{C}_{\text {max }}$ se alcanza entre las 0,25 a $4 \mathrm{~h}$ lo cual concuerda con lo observado en este estudio.

Al observar los valores de AUC es importante destacar la diferencia estadísticamente significativa respecto a ambos esquemas de administración. Entre las razones para que existan diferencias en los valores de AUC experimentales se encuentran factores fisiológicos que modifican la biodisponibilidad de los medicamentos. En este trabajo, los factores más importantes son aquellos que puedan influir sobre el tránsito intestinal como por ejemplo la velocidad del vaciamiento gástrico y el efecto de los alimentos sobre la absorción. Sin embargo, y según lo reportado por Spénard J. y cols ${ }^{18}$, los alimentos producen una leve disminución de la absorción oral de metronidazol, disminuyendo sólo la tasa de absorción pero no afectan la cantidad total de fármaco absorbida. Es decir, la presencia de alimentos no afecta de manera significativa la biodisponibilidad de este antimicrobiano ${ }^{19}$.

Por su parte, destacó la amplia distribución en tejidos y fluidos, observándose valores de $\mathrm{Vd}$ aparente comprendidos entre 0,48 y $0,69 \mathrm{~L}^{*} \mathrm{~kg}^{-1}$, sin existir diferencias significativas respecto a ambos esquemas de administración. Según la bibliografía consultada ${ }^{5}$, el $\mathrm{Vd}$ en adulto 
se encuentra entre $0,5 \mathrm{~L} / \mathrm{kg}$ y $1,1 \mathrm{~L} / \mathrm{kg}$, lo que concuerda con lo establecido en este estudio.

Metronidazol demostró un $\mathrm{t}_{1 / 2}$ comprendido entre 6,1 y 7,5 h. Debido a que la eliminación de metronidazol ocurre principalmente por vía renal, previa metabolización hepática, se podría esperar un incremento de este parámetro en pacientes con una función renal disminuida así como también en adultos de edad avanzada, pudiendo incurrir en una posible acumulación del metronidazol, así como de su metabolito hidroxilado. Adicionalmente, algunos autores señalan que metronidazol posee un $\mathrm{Cl}$ dependiente de la dosis, situación que no se observó en este estudio ${ }^{15}$. Ambas situaciones requieren del análisis individual respecto a las ventajas y posibles riesgos, ya que es importante considerar estos aspectos al recomendar el uso de una dosis superior de metronidazol en pacientes de edad avanzada o con algún grado de disfunción renal. Al respecto, la administración de metronidazol en esquemas tradicionales de dosificación no requiere ajustes de dosis cuando existe disfunción renal, salvo cuando el Clcr es inferior a $30 \mathrm{~mL} / \mathrm{min}$.

Si se considera la distribución de la CIM para $B$. fragilis susceptibles a metronidazol a valores iguales o inferiores a $2 \mathrm{mg} / \mathrm{mL}$, la relación $\mathrm{AUC}_{0-24 \mathrm{~h}} / \mathrm{CIM}$ que predice de mejor forma la efectividad de metronidazol es una relación igual o superior a $70^{11}$. Es decir, con un esquema de administración de $500 \mathrm{mg}$ no sería posible alcanzar esta relación, de tal forma que no sería recomendable para el tratamiento de infecciones graves producidas por anaerobios. Sin embargo, si se considera lo señalado por Isla y cols. ${ }^{10}$, que una relación $\mathrm{PK} / \mathrm{PD}$ mínima para ejercer un efecto terapéutico óptimo de metronidazol es de 25 para infecciones leves en pacientes inmunocompetente, con ambos esquemas sería posible alcanzar un efecto terapéutico deseado. Como se mencionó anteriormente, las diferencias observadas respecto al $\mathrm{AUC}_{0-24 \mathrm{~h}}$ de la administración de $500 \mathrm{mg}$ respecto a los valores descritos en la literatura científica ${ }^{12,16}$ influyen directamente sobre la relación calculada, ya que al establecer una relación $\mathrm{AUC}_{0-24 \mathrm{~h}} / \mathrm{CIM}$ para $B$. fragilis con los valores descritos en la bibliografía, sí es posible alcanzar la meta terapéutica definida. Esto no implica necesariamente que el esquema de $500 \mathrm{mg}$ administrado, así como las formulaciones orales empleadas en el estudio, tengan una biodisponibilidad inferior y que, por tanto, no sean terapéuticamente efectivas en infecciones graves que requieran una relación de PK/PD superior, ya que para el caso de metronidazol, es importante considerar el efecto del metabolito activo sobre B. fragilis. Al respecto, es importante destacar lo señalado por Pendland y cols. ${ }^{20}$, quienes determinaron un efecto bactericida importante para el metabolito el cual exhibe una $\mathrm{CIM}_{90}$ para $B$. fragilis comprendida entre 0,5 y $2,0 \mathrm{mg} / \mathrm{mL}$. Por su parte, metronidazol experimenta metabolismo hepático por un proceso oxidativo, mediado principalmente por actividad catalizada por la enzima CYP2A $6^{21}$ hacia la formación de dos metabolitos principales, el ácido 2-(2-metil-5-nitroimidazol-1-il)-acético el que no posee actividad farmacológica y 1-(2-hidroxietil)2-hidroximetil-5-nitroimidazol (2-hidroximetrondiazol) que contribuye con aproximadamente $30 \%$ de la actividad bactericida de metronidazol ${ }^{22}$. Adicionalmente, el efecto del metabolito activo poseería un efecto sinérgico hacia la actividad de metronidazol; sin embargo, esto último aún no ha sido del todo demostrado.

Numerosos autores han determinado la efectividad de la administración de esquemas abreviados de metronidazol para el manejo de infecciones anaerobias. Al respecto, Hermsen y cols. ${ }^{23}$, compararon la efectividad de un tratamiento asociado con levofloxacina y metronidazol en dosis diaria única versus un tratamiento simple con moxifloxacina. Para ellos, los autores determinaron la relación AUC/CIM de ambos tratamiento sobre cepas de Escherichia coli y B. fragilis en un modelo in vitro. Los autores concluyeron que la administración de un esquema con levofloxacina y metronidazol en esquema tradicional (500 mg cada $8 \mathrm{~h}$ ) y en esquema ampliado ( $1.500 \mathrm{mg}$ cada $24 \mathrm{~h}$ ) produce una actividad bactericida comparable. Por su parte, Wang S. y cols. ${ }^{24}$, realizaron un análisis retrospectivo para comparar la eficacia clínica de un régimen de dosificación en una única administración diaria (1.000 mg cada $24 \mathrm{~h}$ ) versus un tratamiento en dosis diaria múltiple (500 mg cada $8 \mathrm{~h}$ ) para el tratamiento de infecciones pélvicas, intra-abdominales e infecciones de pie diabético producidas por $B$. fragilis, concluyendo que no existen diferencias estadísticamente significativas respecto a la duración de la estadía hospitalaria así como en el grado de compromiso del paciente, y que la administración de un esquema de dosificación en dosis diaria única posee ventajas $\mathrm{PK} / \mathrm{PD}$ para el tratamiento de infecciones graves producidas por B. fragilis. Sin embargo, este estudio es de tipo retrospectivo, en el que no se realizó un análisis PK sobre este esquema de dosificación.

En relación a las probabilidades de alcanzar las metas de AUC/CIM con el esquema abreviado, Isla A. y cols. ${ }^{7,10}$ y Jacob $\mathrm{MR}^{25}$ señalan que, para los derivados 5-nitroimidazólicos, se requiere una relación $\mathrm{AUC}_{0-24 \mathrm{~h}} / \mathrm{CIM}$ superior a 25 para tener éxito en el manejo de infecciones no graves. Por su parte, Sprandel K. y cols. ${ }^{11}$, realizaron una simulación para establecer cuál era la probabilidad de alcanzar la relación AUC/ CIM mayor a 70 tras la administración de tres diferentes esquemas farmacológicos. Los autores determinaron que la administración de un esquema de $500 \mathrm{mg}$ cada 8 h, así como la administración de $1.500 \mathrm{mg}$ cada $24 \mathrm{~h}$, permite alcanzar un meta terapéutica en $99 \%$ de los casos, lo cual no ocurriría con la administración de $1.000 \mathrm{mg}$ cada $24 \mathrm{~h}$ donde solamente se alcanza en $72 \%$. Es importante señalar que en este estudio, el autor considera para la simulación 
de los datos un comportamiento bi compartimental para metronidazol además de evaluar el efecto del metabolito hidroxilado. Estas son diferencias claras respecto a los resultados determinados en nuestro trabajo las cuales requieren de nuevos estudios.

\section{Conclusión}

Desde el punto de vista farmacocinético y farmacodinámico, con la administración de un esquema de metronidazol en una dosificación de $1.000 \mathrm{mg}$ cada $24 \mathrm{~h}$ para el manejo de infecciones producidas por cepas de $B$. fragilis sensibles a metronidazol, sería posible alcanzar una relación $\mathrm{AUC}_{0-24 \mathrm{~h}} / \mathrm{CIM}$ superior al esquema de dosificación de $500 \mathrm{mg}$ cada $8 \mathrm{~h}$. De tal forma que es posible considerar la administración de una dosis diaria de $1.000 \mathrm{mg}$ de metronidazol como una opción terapéutica al esquema tradicional. Sin embargo, es necesario considerar la variabilidad interindividual, y comprobar en la práctica clínica que este esquema sea terapéuticamente efectivo y seguro.

\section{Limitaciones}

Es importante hacer hincapié en el carácter experimental de este estudio realizado con un reducido número de voluntarios. De tal manera que la mayoría de las sus sugerencias son de carácter teórica con parámetros simulados a partir de datos experimentales, por lo cual no constituyen una recomendación para su aplicación clínica ya que es necesario establecer el comportamiento del esquema de dosificación sugerido en condiciones clínicas reales.

\section{Resumen}

Introducción: Metronidazol es el antimicrobiano de elección para el manejo de infecciones anaeróbicas. Su administración requiere de dosis múltiples provocando aumento en errores medicamentosos. Debido al efecto post-antibiótico y a la actividad bactericida concentración-dependiente, la administración de metronidazol en intervalos ampliados de administración permitiría alcanzar parámetros $\mathrm{PK} / \mathrm{PD}$ efectivos. Objetivo: Evaluar la probabilidad de alcanzar una relación PK/PD efectiva con la administración de $1.000 \mathrm{mg}$ cada $24 \mathrm{~h}$ de metronidazol para infecciones por Bacteroides fragilis. Método: Se realizó un ensayo clínico sobre un grupo de voluntarios a quienes se les administró una monodosis oral de 500 y $1.000 \mathrm{mg}$ de metronidazol, respectivamente. Se establecieron parámetros farmacocinéticos empleando el método trapezoidal. Se realizó una simulación de Markov que permitiera establecer la probabilidad de alcanzar una relación $\mathrm{AUC}_{0-24 \mathrm{~h}} / \mathrm{CIM}>70$ en infecciones por B. fragilis. Resultados: Se determinaron los valores $\mathrm{de}_{\max }(24,03 \pm 6,89 \mathrm{mg} / \mathrm{L}), \mathrm{t}_{\max }(1,20 \pm 0,8 \mathrm{~h})$ y $\mathrm{AUC}_{0-24 \mathrm{~h}}$ $(241,91 \pm 48,14 \mathrm{mg} * \mathrm{~h} / \mathrm{L})$, con lo cual la probabilidad de alcanzar una relación $\mathrm{AUC}_{0-24 \mathrm{~h}} / \mathrm{CIM}>70$ con $1.000 \mathrm{mg}$ de metronidazol fue superior a 99\%. Conclusión: Con la administración de $1.000 \mathrm{mg}$ cada 24 h sería posible alcanzar una relación PK/PD efectiva para el tratamiento de infecciones anaeróbicas.

\section{Referencias billbiográficas}

1.- Lassmann B, Gustafson D R, Wood C M, Rosenblatt J E. Reemergence of anaerobic bacteremia. Clin Infect Dis 2007; 44 (7): 895-900.

2.- Fenner L, Widmer A F, Straub C, Frei R. Is the incidence of anaerobic bacteremia decreasing? Analysis of 114,000 blood cultures over a ten-year period. J Clin Microbiol 2008; 46 (7): 2432-34.

3.- Mandell GLBJEDR. Mandell, Douglas, and Bennett's Principles and Practice of Infectious Diseases. Philadelphia, PA: Churchill Livingstone/Elsevier, 2010

4.- Solomkin J S, Mazuski J E, Bradley J S, Rodvold K A, Goldstein E J C, Baron E J, et al. Diagnosis and management of complicated intra-abdominal infection in adults and children: Guidelines by the Surgical Infection Society and the Infectious Diseases Society of America. Clin Infect Dis 2010; 50 (2): 133-64.
5.- Lamp K C, Freeman C D, Klutman N E, Lacy M K. Pharmacokinetics and pharmacodynamics of the nitroimidazole antimicrobials. Clin Pharmacokinet 1999; 36 (5): 353-73.

6.- Estes L. Review of pharmacokinetics and pharmacodynamics of antimicrobial agents. Mayo Clinic Proc 1998;73 (11): 1114-22.

7.- Isla A, Canut A, Rodríguez-Gascón A, Planells P, Beltrí-Orta P, Salmerón-Escobar J I, et al. Utilización de antimicrobianos en las infecciones odontogénicas en niños y adolescentes: análisis farmacocinético/ farmacodinámico (PK/PD). Enferm Infecc Microbiol Clin 2008; 26 (10): 621-8.

8.- do Nascimento T G, de Jesus Oliveira E, Macêdo R O. Simultaneous determination of ranitidine and metronidazole in human plasma using high performance liquid chromatography with diode array detection. J Pharm Biomed Anal 2005; 37 (4): 777-83.

9.- Diseases ESoCMaI. The European Committee on Antimicrobial Susceptibility Testing EUCAST: http://www.eucast.org/, 2010.

10.- Isla A, Canut $A$, Rodríguez-Gascón $A$, Labora A, Ardanza-Trevijano B, Solinís M Á, et al. Análisis farmacocinético/farmacodinámico (PK/PD) de la antibioterapia en odontoestomatología. Enferm Infecc Microbiol Clin 2005; 23(3): 116-21.

11.- Sprandel K A, Drusano G L, Hecht D W, Rotschafer J C, Danziger L H, Rodvold K A. Population pharmacokinetic modeling and Monte Carlo simulation of varying doses of intravenous metronidazole. Diagn Microbiol Infect Dis 2006; 55 (4): 303-9.

12.- de C Bergamaschi C, Berto L A, Venâncio P C, Cogo K, Franz-Montan M, Motta R H L, et al. Concentrations of metronidazole in human plasma and saliva after tablet or gel administration. J. Pharm. Pharmacol 2014; 66 (1): 40-7.

13.- Amon I, Amon K, Hüller H. Pharmacokinetics and therapeutic efficacy of metronidazole at 
different dosages. Int J Clin Pharmacol 1978; 16: 384-6.

14.- Bergan T, Arnold E. Pharmacokinetics of metronidazole in healthy volunteers after tablets and suppositories. Chemotherapy 1980; 26: 231-41.

15.- Freeman C, Klutman N, Lamp K. Metronidazole. Drugs 1997; 54 (5): 679-708.

16.- Montalli V A, de Cassia Bergamaschi C, Ramacciato J C, Nolasco F P, Groppo F C, de Brito R B, et al. The effect of smoking on the bioavailability of metronidazole in plasma and saliva. J Am Dent Assoc 2012; 143 (2): 149-56.

17.- de C Bergamaschi C, Berto L A, Venâncio P C, Cogo K, Franz-Montan M, Motta R H L, et al. Concentrations of metronidazole in human plasma and saliva after tablet or gel administration. J Pharm Sci 2014; 66 (1): 40-7.

18.- Spénard J, Aumais C, Massicotte J, Brunet J-S, Tremblay C, Grace M, et al. Effects of food and formulation on the relative bioavailability of bismuth biskalcitrate, metronidazole, and tetracycline given for Helicobacter pylori eradication. Br. J. Clin. Pharmacol 2005; 60 (4): 374-77.

19.- Lee L L, Rowland J, Ling C H Y, Fiakos E. A quantity survey of intravenous administration of metronidazole in its different forms in a tertiary teaching hospital. Intern Med J 2010; 40 (8): 592-6.

20.- Pendland S L, Piscitelli S C, Schreckenberger P C, Danziger L H. In vitro activities of metronidazole and its hydroxy metabolite against Bacteroides spp. Antimicrob. Agents Chemother 1994; 38 (9): 2106-10.

21.- Pearce R E, Cohen-Wolkowiez M, Sampson M R, Kearns G L. The role of human cytochrome p450 enzymes in the formation of 2-hydroxymetronidazole: CYP2A6 is the high affinity (low km) catalyst (Abstract). Drug Metab Dispos 2013; 41 (9): 1686-94.

22.- Jessa M J, Barrett D A, Shaw P N, Spiller R C. Rapid and selective high-performance liquid chromatographic method for the determination of metronidazole and its active metabolite in human plasma, saliva and gastric juice. J Chromatogr B Biomed Sci Appl 1996; 677 (2): 374-9.

23.- Hermsen E D, Hovde L B, Sprandel K A, Rodvold K A, Rotschafer J C. Levofloxacin plus metronidazole administered once daily versus moxifloxacin monotherapy against a mixed infection of Escherichia coli and Bacteroides fragilis in an in vitro pharmacodynamic model. Antimicrob. Agents Chemother 2005; 49 (2): 685-9.

24.- Wang S, Cunha B A, Hamid N S, Amato B M, Feuerman M, Malone B. Metronidazole single versus multiple daily dosing in serious intraabdominal/pelvic and diabetic foot infections. J Chemother 2007; 19 (4): 410-6.

25.- Jacobs M R. Optimisation of antimicrobial therapy using pharmacokinetic and pharmacodynamic parameters. Clin Microbiol Infec 2001; 7(11): 589-96. 